\title{
Non-pharmacological Community Intervention, Especially Pain Management, in Rheumatoid Arthritis: A Review of the Literature \\ H Yousefi ${ }^{1}$, A Chopra ${ }^{2}$, R Farrokhseresht ${ }^{3}$, FA Noghabi ${ }^{1}$, N Kulkarni $^{2}$
}

\begin{abstract}
Objective: Rheumatoid arthritis (RA) is a crippling disease with significant affection in a patient's life. The objective of this study is to describe the role of uni-modular and multi-modular non-pharmacological community intervention effectiveness, especially pain management interventions in rheumatoid arthritis.

Methods: This review built on a preliminary literature search, covering 2009 up to December 2013. Selective review of current literature was produced by searching the term nonpharmacological intervention, "self-management programme", "self-care", "rheumatoid arthritis" to capture all spectrums of rheumatoid arthritis non-pharmacological intervention. Twenty-six reviews were included in this overview.

Results: A substantial and remarkable number of studies of non-drug care interventions in RA are available. Twenty-six reviews were included in the present overview indicated a beneficial effect of cognitive-behavioural therapy and psychotherapeutic intervention, self-management, physical therapy (Exercise) but a few studies indicated a beneficial effect of the multidisciplinary education programme and specific dietary interventions. The evidence of effectiveness varies among the different non-pharmacological modalities and indicates a need for further investigation into the most clinically and cost-effective strategies to deliver individual, nonpharmacological treatment modalities as well as comprehensive arthritis service delivery models for RA patients.
\end{abstract}

Conclusion: This review gives a summary of the available evidence regarding the effectiveness of non-pharmacological treatment modalities are often prescribed as an adjunct to standard care in RA, but the data need scientific appraisal into the most clinically and cost-effective strategies

Keywords: Intervention studies, non-drug therapy, pain management, rheumatoid arthritis

From: ${ }^{1}$ Faculty of Nursing, Midwifery and Paramedical, Hormozgan University of Medical Sciences, Bandar Abbas, Hormozgan, Iran, ${ }^{2}$ Center for Rheumatic Diseases, Savitribai Phule Pune University, Pune, India and ${ }^{3}$ Department of Internal Medicine, Faculty of medicine, Hormozgan University of Medical Sciences, Bandar Abbas, Hormozgan, Iran.

Correspondence: Dr H Yousefi, Department of Health Sciences, Faculty of Nursing, Midwifery and Paramedical, Hormozgan University of Medical Sciences, Bandar Abbas, Hormozgan, Iran. Tel: 00989171594097.

E-mail: hadiyousefi1@gmail.com

West Indian Med J 2016; 65

DOI: 10.7727/wimj.2015.468 


\section{INTRODUCTION}

The spectrum and severity of rheumatoid arthritis (RA) is not very different in developing and developed countries, the dismal rheumatology services in developing countries further compounds the burden (1). The incidence and prevalence of these conditions, it has been proved, is dynamic, not static, and appears to be influenced by both genetic and environmental factors (2). Ample information has been collected in the community oriented programme for control of rheumatic disease (COPCORD) studies (3) to show wide prevalence of RA. The prevalence of clinical RA recently reported by the WHO ILAR COPCORD urban and rural surveys in India varied from 0.45 to $0.68 \%$ (4) in Iran was noted as urban $0.33 \%$ and rural $0.19 \%(5,6)$ in Asia Pacific countries was calculated $0.33 \%$ (7) and in developed populations $0.5 \%$ to $1 \%$ of the adult population (8).

Despite substantial advances in medical treatment, RA is a disease that continues to affect the lives of individuals considerably $(9,10)$. The challenge is to find a cost-effective treatment for better disease control $(11,12)$. Pharmacological treatment has recently seen great advances, but is associated with increased toxicity and cost also the long term outcome is still unknown. Non-pharmacological treatment is cheap, less toxicity better long term outcome $(4,9,10,13)$.

The objective of this literature review is to present the most recent evidence related to the non-pharmacological intervention programme for management of the rheumatoid arthritis to inform the development of evidence based recommendations for general practitioners working in the healthcare setting. 


\section{MATERIAL AND METHODS}

This review is built on a preliminary literature search. The literature review was conducted in July 2010 up to 2014 . Articles published from 1990 to 2013 were considered. The literature review was raised by a thorough search conducted in MEDLINE, Pub MED (Pub MED Central and pub MED Heath), EMBASE, Web of Science and the Cochrane Library were searched to identify studies for inclusion.

The following search strategies were adapted to apply to the other databases. Using a MESH term of "rheumatoid arthritis", "arthritis", "rheumatic diseases", "Joint", "complementary therapies", "non-prescription drugs", "intervention studies", "self-care", "pain management" to capture all spectrums of rheumatoid arthritis non-pharmacological intervention and selfmanagement practices, inclusion was limited to English language publications. The final search strategy sought to identify non-pharmacological intervention studies of all levels of evidence. Concurrently a manual search was carried out to find an article review of reference lists of retrieved papers. In the beginning, the papers were selected for inclusion based on the title and abstract. Studies providing evidence on the efficacy of an intervention or compared to another intervention were included. Initial searches failed to identify many articles related to nonpharmacological intervention programme for management of the rheumatoid arthritis.

Participants of interest to this literature review were people aged 16 years or over with a diagnosis of RA. The review focussed on data in adult populations (above 16 years of age). Intervention in the form of any non-pharmacological intervention used to manage RA was eligible for inclusion. Articles focussing on non-pharmacological interventions in RA, with a 
community approach, big sample size, with control, randomize control trial (RCT) and having a core measure of pain VAS were selected. Other alternative treatments controversy has arisen over the employment of therapies same occupational therapy, podiatry, hydrotherapy, joint protection, ultrasound, acupuncture, laser therapy, use of compression gloves, thermotherapy, use of splints or orthoses, homeopathy and transcutaneous electrical nerve stimulation (TENS) which were not being eligible for inclusion.

The search strategy was formulated by Ovid in cooperation with a medical librarian to make it applicable to all the databases. A computerized broad search strategy was developed. Retrieved hits were assessed by three of the authors (HY, FA, NK), who screened the titles and abstracts to identify relevant studies. If there was doubt about a study's relevance, one of the expert authors (AC) was consulted. The relevant full-text article was read by three authors (AC, HY, RF). The methodological quality of included review was independently assessed by two reviewers (AC, HY). Data was extracted by two of the authors (HY, NK). If doubt occurred, one of the other authors (AC) was consulted. In the search finding following statement was used to indicate direction of reduced pain effect.

\section{RESULTS}

The literature search identified 14362 references, which were first examined on the basis of titles and abstracts. Of these, 13963 references were not related to the non-pharmacological intervention programme for management of the rheumatoid arthritis diseases. Two hundred and 
sixty-one references were not RCT study. One hundred and thirty-eight references were retrieved in full text. One hundred and twelve reviews were excluded: 49 because of conducted to mix rheumatic and musculoskeletal disease and 41 were conducted by other than rheumatoid arthritis. 19 because of no relevant intervention on pain. Three because of duplicate publication. Twentysix reviews were included in this overview (Fig. 1).

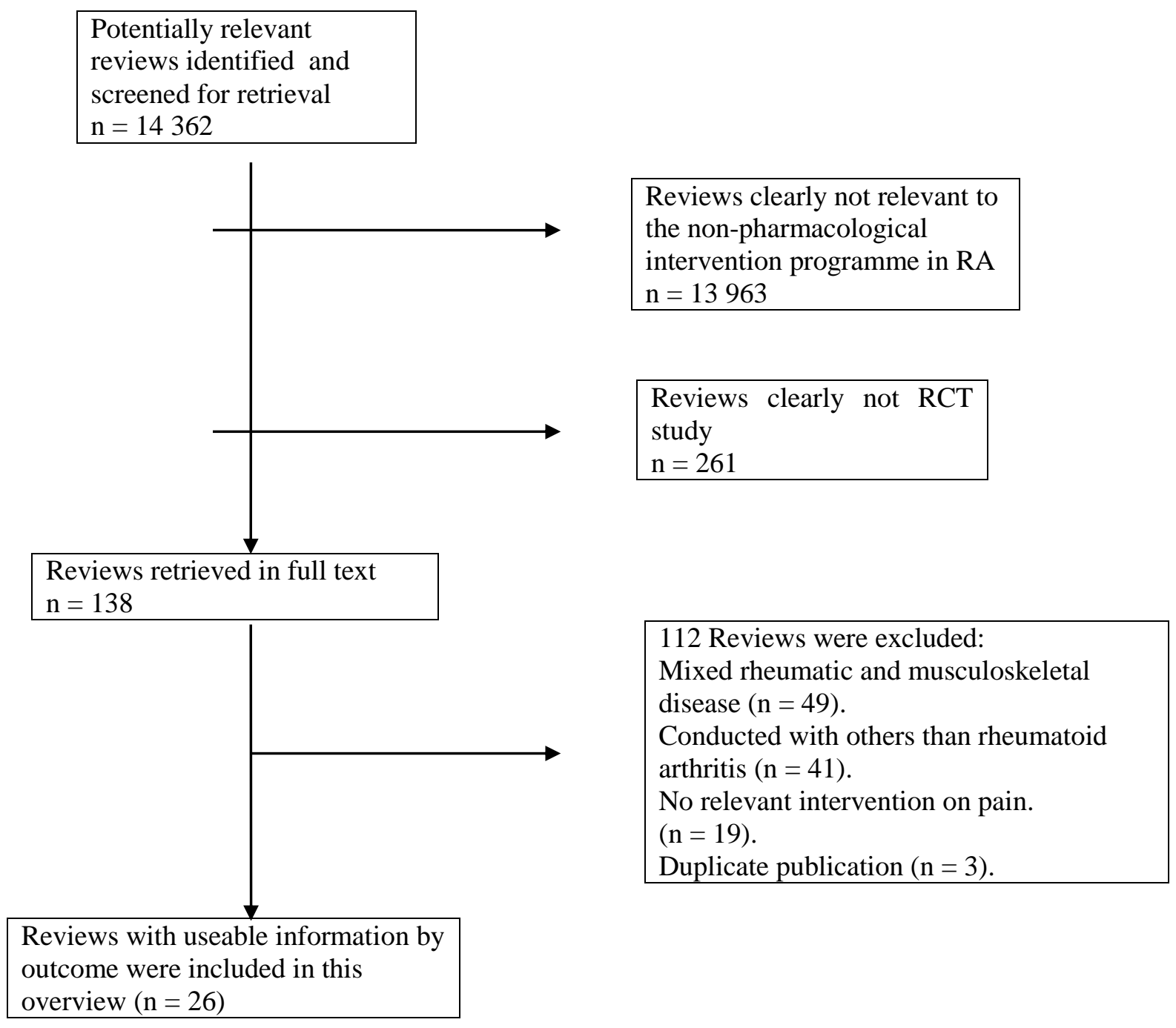

Fig: 1. Selection process of eligibility reviews from all identified citations. 


\section{Cognitive-behavioural therapy and psychotherapeutic intervention}

A hospital based on six month studies by Sharp et al, designed and conducted to examine the efficacy of CBT (Fig. 2) on preventing psychological and physical morbidity in patients with RA.

Fig: 2. Uni-modular and multi-modular self-management programme

CBT: Included 8 individual sessions each of 1hour per week, developed from standard pain management approaches and selfhelp educational material developed for patients with arthritis. The programme included an educational component plus the self-management skills of relaxation training, attention diversion, goal setting, pacing, problem-solving, cognitive restructuring, assertiveness and communication, and management of flare-ups or high-risk situations [14].

LMAP: Included two modules, each with four meetings of 2.5 hours duration and one 2-hours review meeting (one rheumatology OT, one community OT and one rheumatology PT delivered modules). Participants could attend two LMAP modules and review meeting over a 3- to 9-month period as convenient to them. Module 1 was developed from a behavioural joint protection programme. The concepts discussed in meeting 1 were RA, health beliefs, personal impact of arthritis, understanding the multiple factors affecting symptoms, attitudes, personal experiences, self-management methods and motivating for change. Meetings 2-4 focussed on ergonomic approaches to reduce pain, exercises, fatigue management and the benefits of splints. Module 2, focussed on participants' exercise beliefs, barriers and problem-solving. Cognitive symptom management included stress management and coping, pain management, distraction and relaxation practice. The review meeting included progress with goals, drug therapy, investigations, communicating with health professionals, teamcare and topics of participants' choice (eg diet, complementary therapies, work support services, Social Security Benefits). Participant workbooks were provided for each module with key points, illustrations and diaries to record practicing joint protection, pacing and exercise [15].

GESO: The programme consisted of 5 weekly 2-hour group sessions for 8 patients, with or without a significant other. The programme included 3 booster sessions of 2 hours each after 3, 6, and 9 months. Each group had 2 trained leaders (during the 2 days of training): a specialized arthritis nurse and a nurse with experience in working with RA patients. Patients received a programme book with information on the sessions, a self-help guide, various brochures on RA, and an audiotape with relaxation exercises. The programme included the contracting, goal setting, self-management and problem solving, Information on RA and treatment, Pain management and relaxation, physical exercises, coping with depression. In the booster sessions, the accomplishments of goals and problems during the past 3 months were discussed and feedback was given. The assessments were done before, immediately after and 6 and 12 months after intervention [18].

MSCG: In the programme patients, who participated in 10 sessions, supported by a manual for patients and led by 2 supervisors. All sessions lasted 2 hours. The first 8 sessions were weekly sessions, the $9^{\text {th }}$ session was 2 weeks after the $8^{\text {th }}$ session, and the $10^{\text {th }}$ session was 3 weeks after the $9 \mathrm{t}^{\mathrm{h}}$ session. At the end of every session (except for the very first session), homework assignments were given. In the CIG, the programme was teaching patients action-directed coping and coping by seeking social support; 4 steps of problem-solving steps: 1) describe the problem; 2) think about all kinds of possible solutions; 3) choose 1 or more solutions; 4) implement the solution or solutions and evaluate the results. In the MSCG programme topics 
of conversation for all sessions were determined by the patients during the first session to exchange information, experiences, feelings, and emotions of the participants. The sessions were led by 2 patients who were trained in supervising mutual support groups [19].

In-patient treatment consisted of a fixed period of 11 days, each session weekly, performed by the physical therapist, occupational therapist and social worker, which prescribed regimens of bed rest and a daily individual range of motion, musclestrengthening exercise programme, joint protection, self-care, household and work activities, joint splints, adaptive equipment and coping with the disease. During out-patient care, the prescription of drugs, paramedical treatment and splints was left to the attending physician to the out-patient clinic [21, 22]. The training programme was scheduled for nine sessions within 2 weeks, each group consisted of eight patients, and encompassed a multidisciplinary cooperation between rheumatologists, orthopedists, physiotherapists, psychologists, and social workers. The following fields were covered: mechanisms of RA, drug therapy, physiotherapy, practical exercise, relieving pain and muscle tension, joint protection devices, joint replacement, coping strategies, stress management and relaxation exercise, dietetics, social assistance and utilization of public social resources [23].

ASMP: It is a 6-week series of classes for 2 to 2.5 hours per session (total 12 hours) included information about arthritis, selfmanagement principles, exercise, cognitive symptom management, relaxation, energy-saving techniques, cognitive pain management, dealing with depression, nutrition, communication with family and health professionals, and contracting [24].

SMART: Mailed intervention is a "tailored print intervention" includes a 1-page questionnaire asking questions about pain disability, exercise levels, and other arthritis-related behaviours. Participants also receive a copy of the Arthritis help book and quarterly follow-up materials. These included a second book, arthritis: a comprehensive guide, a relaxation tape, and a pamphlet on physician/patient communications. The sequence of the questionnaire, letter, and report deliverable is repeated every 4 months for 1 year. The result showed that there was an improvement in all baseline variables for SMART and ASMP groups with higher benefit in self-efficacy and doctor visit rate [22]

CDSMP: It is community based, a 2.5 hours lecture per week over a 6 week period included healthy eating, starting and maintaining exercise, pain and fatigue management, managing sleep, stress management, relaxation techniques, communicating with health providers, managing medications planning and problem solving [29].

The results indicate efficacy in producing reductions in both psychological and physical morbidity (14) [Table 1]. Another study conducted by the same groups over 18 months revealed that the capacity of coping with pain and depression improved, but no change was observed in other variables (15) [Table 1]. 
Table 1: Uni-modular self-management programme in rheumatoid arthritis

\begin{tabular}{|c|c|c|c|c|c|c|c|c|c|}
\hline Year & Name of study & $\begin{array}{c}\text { Sample } \\
\text { size }\end{array}$ & Design & $\begin{array}{c}\text { Durati } \\
\text { on of } \\
\text { study }\end{array}$ & $\begin{array}{c}\text { Duration of } \\
\text { RA }\end{array}$ & Intervention & $\begin{array}{c}\text { Key efficacy } \\
\text { variable }\end{array}$ & Key finding & Reference \\
\hline 1993-1994 & $\begin{array}{l}\text { Vegetarian diet } \\
\text { on clinical } \\
\text { effect of } \\
\text { psychological } \\
\text { characteristics }\end{array}$ & 124 & $\begin{array}{l}\text { RCT; } \\
3 \text { arms: Vegetarian diet } \\
\text { Omnivorous diet } \\
\text { and control groups } \\
\text { site: Hospital based } \\
\text { rheumatology }\end{array}$ & $\begin{array}{c}13 \\
\text { months }\end{array}$ & $\begin{array}{l}\text { Mean } 13 \text { years } \\
\text { intervention } \\
\text { and } 10 \text { years } \\
\text { controls }\end{array}$ & $\begin{array}{l}\text { Vegetarian } \\
\text { diet } \\
\text { Omnivorous } \\
\text { diet }\end{array}$ & $\begin{array}{l}\text { GHQ-20, } \\
\text { MHLCS, } \\
\text { Pain VAS, ESR, } \\
\text { TJC, TJS } \\
\text { Believe in } \\
\text { Ordinary and } \\
\text { alternative } \\
\text { treatment VAS }\end{array}$ & $\begin{array}{l}\text { GHQ(anxiety), } \\
\text { Internal MHLCS, } \\
\text { Believe in } \\
\text { Ordinary and } \\
\text { alternative } \\
\text { significant } \\
\text { improvement } \\
\text { and psychological } \\
\text { distress decreased }\end{array}$ & $\begin{array}{l}\text { Kjeldsen- } \\
\text { Kragh et al } \\
(1994)^{34}\end{array}$ \\
\hline 1997-1998 & $\begin{array}{l}\text { effects of } \\
\text { uncooked vegan } \\
\text { diet, rich in } \\
\text { lactobacilli, in } \\
\text { RA }\end{array}$ & 43 & $\begin{array}{l}\text { RCT; } \\
2 \text { arms: } \\
\text { Vegetarian diet } \\
\text { and control groups } \\
\text { site: Hospital based } \\
\text { rheumatology }\end{array}$ & $\begin{array}{c}6 \\
\text { months }\end{array}$ & $\begin{array}{l}\text { Mean } \\
12.6 \pm 12.3 \\
\text { years in } \\
\text { intervention } \\
\text { and } 16.1 \pm 13.6 \\
\text { years in } \\
\text { control groups }\end{array}$ & $\begin{array}{l}\text { uncooked } \\
\text { vegan diet, } \\
\text { rich in } \\
\text { lactobacilli }\end{array}$ & $\begin{array}{l}\text { DAS28, TJC, TJS } \\
\text { morning } \\
\text { stiffness } \\
\text { protein } \\
\text { (S-CRP), } \\
\text { pain VAS, } \\
\text { HAQ and } \\
\text { global patient }\end{array}$ & $\begin{array}{l}\text { lost weight, pain } \\
\text { VAS, DAS-28, } \\
\text { HAQ } \\
\text { Disease activity } \\
\text { Improved }\end{array}$ & $\begin{array}{l}\text { Nenonen } \\
\text { et al } \\
(1998)^{35}\end{array}$ \\
\hline $\begin{array}{c}1993 \text { to } \\
1995\end{array}$ & $\begin{array}{l}\text { Efficacy of } \\
\text { physical therapy }\end{array}$ & 127 & $\begin{array}{l}\text { Single- blind RCT; } \\
2 \text { arms: } \\
\text { control wait list; } \\
\text { intervention care; } \\
\text { site: Community based } \\
\text { rheumatology }\end{array}$ & 2 years & $\begin{array}{l}\text { mean } \\
7.6 \pm 11 \text { and } \\
7.4 \pm 10 \text { in } \\
\text { experimental } \\
\text { and control } \\
\text { group } \\
\text { respectively }\end{array}$ & $\begin{array}{l}\text { nutrition, } \\
\text { exercise, } \\
\text { background } \\
\text { Std care (RA) }\end{array}$ & $\begin{array}{l}\text { Self-efficacy, } \\
\text { pain VAS, } \\
\text { disease activity, } \\
\text { duration of } \\
\text { morning } \\
\text { stiffness, } \\
\text { tender joint count }\end{array}$ & $\begin{array}{l}\text { Improvement in all } \\
\text { variables except pain } \\
\text { and disease activity. }\end{array}$ & $\begin{array}{l}\text { Bell et al } \\
\qquad(1998)^{33}\end{array}$ \\
\hline
\end{tabular}




\begin{tabular}{|c|c|c|c|c|c|c|c|c|c|}
\hline $1994-1996$ & $\begin{array}{l}\text { Cognitive } \\
\text { behaviour } \\
\text { intervention on } \\
\text { preventing } \\
\text { psychological } \\
\text { and physical } \\
\text { morbidity }\end{array}$ & 53 & $\begin{array}{l}\text { Single blind (assessor) } \\
\text { RCT; } \\
2 \text { arms: control; } \\
\text { intervention care; } \\
\text { Site: Hospital based } \\
\text { clinical Psychologist }\end{array}$ & $\begin{array}{c}6 \\
\text { months }\end{array}$ & $\begin{array}{l}\text { less than } 2 \\
\text { years }\end{array}$ & $\begin{array}{l}\text { cognitive } \\
\text { behaviour } \\
\text { therapy, } \\
\text { background } \\
\text { Std care }\end{array}$ & $\begin{array}{l}\text { HADS, HAQ, } \\
\text { pain VAS }\end{array}$ & $\begin{array}{l}\text { HADS, depression } \\
\text { ad anxiety, HAQ } \\
\text { showed } \\
\text { significant } \\
\text { improvement but no } \\
\text { benefit in pain }\end{array}$ & $\begin{array}{l}\text { Sharp et al } \\
(2001)^{14}\end{array}$ \\
\hline 2001 & $\begin{array}{l}\text { Effect of patient } \\
\text { education } \\
\text { programme on } \\
\text { adherence to drug } \\
\text { treatment }\end{array}$ & 100 & $\begin{array}{l}\text { Single blind (assessor) } \\
\text { RCT, } \\
2 \text { arms: control; } \\
\text { intervention care; } \\
\text { site: Hospital based } \\
\text { clinical pharmacology }\end{array}$ & $\begin{array}{c}6 \\
\text { months }\end{array}$ & median 12 year & $\begin{array}{l}\text { patient } \\
\text { education } \\
\text { programme, } \\
\text { background } \\
\text { Std care (RA) }\end{array}$ & $\begin{array}{l}\text { articular index, } \\
\text { morning } \\
\text { stiffness, } \\
\text { pain VAS }\end{array}$ & $\begin{array}{l}\text { improvement in drug } \\
\text { adherence but no } \\
\text { clinical benefit }\end{array}$ & $\begin{array}{l}\text { Hill et al } \\
(2001)^{19}\end{array}$ \\
\hline 2001 & $\begin{array}{l}\text { A vegan diet free } \\
\text { of gluten } \\
\text { improves the } \\
\text { signs and } \\
\text { symptoms of } \\
\text { rheumatoid } \\
\text { arthritis }\end{array}$ & 66 & $\begin{array}{l}\text { RCT } \\
2 \text { Arms: } \\
\text { vegan diet } \\
\text { and non-vegan diet } \\
\text { site: Hospital based } \\
\text { rheumatology }\end{array}$ & 1 year & $\begin{array}{l}\text { Mean } 5.2 \pm 2.5 \\
\text { years in } \\
\text { intervention } \\
\text { and } 5.8 \pm 2.8 \\
\text { years in } \\
\text { control groups }\end{array}$ & $\begin{array}{l}\text { a vegan diet } \\
\text { free of gluten } \\
\text { and a } \\
\text { well-balanced } \\
\text { non-vegan diet }\end{array}$ & $\begin{array}{l}\text { IgG, } \\
\beta \text {-lactoglobulin } \\
\text { X-Ray } \\
\text { TJC, TJS, CRP, } \\
\text { PhGA }\end{array}$ & $\begin{array}{l}\text { significant } \\
\text { improvement in } \\
\text { clinical parameters, } \\
\text { IgG, } \beta \text {-lactoglobulin } \\
\text { level decreased } \\
\text { no significant } \\
\text { differences in X-Ray }\end{array}$ & $\begin{array}{l}\text { Hafström } \\
\text { et al } \\
(2001)^{36}\end{array}$ \\
\hline $1994-1996$ & $\begin{array}{l}\text { Long-term } \\
\text { efficacy of } \\
\text { cognitive } \\
\text { behaviour } \\
\text { therapy }\end{array}$ & 53 & $\begin{array}{l}\text { Single blind (assessor) } \\
\text { RCT } \\
2 \text { arms: control; } \\
\text { intervention care; } \\
\text { site: Hospital based } \\
\text { clinical Psychology }\end{array}$ & $\begin{array}{c}18 \\
\text { months }\end{array}$ & $\begin{array}{l}\text { less than } 2 \\
\text { years }\end{array}$ & $\begin{array}{l}\text { cognitive } \\
\text { behaviour } \\
\text { programme } \\
\text { (CBP), } \\
\text { background } \\
\text { Std care (RA) }\end{array}$ & $\begin{array}{l}\text { HADS, HAQ, } \\
\text { pain, VAS, } \\
\text { coping, } \\
\text { Ritchie articular } \\
\text { index }\end{array}$ & $\begin{array}{l}\text { coping and } \\
\text { depression improved } \\
\text { but no change in } \\
\text { other variables }\end{array}$ & $\begin{array}{l}\text { Sharp et al } \\
(2003)^{15}\end{array}$ \\
\hline $2004-2005$ & $\begin{array}{l}\text { Mediterranean } \\
\text { dietary on Fat } \\
\text { intake and } \\
\text { composition of } \\
\text { fatty acids in } \\
\text { serum } \\
\text { phospholipids }\end{array}$ & 51 & $\begin{array}{l}\text { RCT (assessor blind) } \\
2 \text { arms: control; } \\
\text { Dietary intervention; } \\
\text { site: Community based } \\
\text { rheumatology }\end{array}$ & $\begin{array}{c}3 \\
\text { months }\end{array}$ & NA & $\begin{array}{l}\text { Mediterranean } \\
\text { dietary: } \\
\text { advised to } \\
\text { replace high } \\
\text { fat dairy } \\
\text { products with } \\
\text { low fat } \\
\text { products }\end{array}$ & $\begin{array}{l}\text { Fat, Total } \\
\text { saturated fatty } \\
\text { acids , } \\
\text { monounsaturated } \\
\text { fatty acids, } \\
\text { polyunsaturated } \\
\text { fatty acids, } \\
\text { n-6 fatty acids, } \\
\text { n-3 fatty acids }\end{array}$ & $\begin{array}{l}\text { a lower ratio of } \\
\text { n- } 6 \text { to n- } 3 \text { fatty acids } \\
\text { better clinical } \\
\text { improvement, higher } \\
\text { intake of n- } 3 \text { fatty } \\
\text { acids and a lower } \\
\text { ratio of n- } 6 \text { to n-3 } \\
\text { fatty acids }\end{array}$ & $\begin{array}{l}\text { Hagfors et al } \\
\quad(2005)^{37}\end{array}$ \\
\hline
\end{tabular}




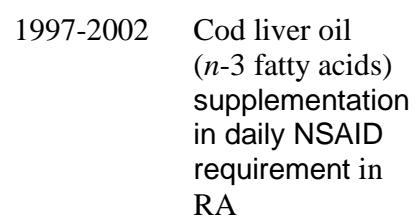

2007-2008 Vegan diet on blood lipids (oxLDL) and

$$
\text { natural }
$$

atheroprotective

(anti- PCs).

$\begin{array}{ll}\text { 2007-2011 } & \text { Internal family } \\ & \text { System based } \\ & \text { psychotherapeuti } \\ & \text { c Intervention } \\ & (\text { IFS) }\end{array}$

97

RCT double-blind
placebo-controlled
2 arms: Vegetarian diet
and control groups
site: Hospital based
rheumatology

66

RCT;

2 arms: vegan diet and control non- vegan diet

site: Community based

rheumatology

79

mont

9
months

Mean $13 \pm 1.26$ intervention and $13 \pm 1.4$ years in control groups

filled identical placebo

capsules.
1 years Mean 5 years vegan group and 5.8 years controls

Vvegan diet
free of gluten
IFS model
intervention,
background
Std care (RA)

intervention care;

Site: Hospital base
Rheumatology

\section{CRP, TJC, TJS \\ DAS-28, Pain}

VAS

HAQ, IgM, EMS

Daily NSAID

requirement
BMI, DAS28,

HAQ,

biochemical

variables,

OxLDL, anti-

PCs Drugs

\section{DAS28-CRP4,}

pain VAS,

depression,

anxiety,

physical

function,
Significantly reduce daily NSAID

requirement

no significant

differences in the

clinical parameters

of RA disease

activity

Decreased LDL and oxLDL levels and raised anti-PC

IgM and IgA levels.

disease activity and medication use unchanged, benefit seen in all other variables.
Galarraga et al $(2008)^{38}$ medication usage

oxLDL: oxidized low-density lipoprotein; anti- PCs: antibodies against phosphorylcholine; BMI: body mass index; LDL: low-density lipoprotein; IgM: immunoglobulin M; MHLCS: Multi-dimensional health locus of control scale; GHQ-20: General Health Questionnaire; EMS: early morning stiffness; IgG:immunoglobulin G; PhGA: physician global assessment; CRP:C-reactive protein.

A longitudinal study was conducted by Hammond et al to develop a modular behavioural group programme "The lifestyle management for arthritis programme" (LMAP) [Fig. 2] and evaluate its longer term effects on pain, physical and psychological status. The study finding indicated that behavioural group continued to have decreased pain and fatigue; better functional ability, psychological status and self-efficacy scores; and greater use of health behaviours (16) [Table 2]. 
Table 2: Multi-modular self-management programme in rheumatoid arthritis

\begin{tabular}{|c|c|c|c|c|c|c|c|c|c|}
\hline Year & Name of study & $\begin{array}{c}\text { Sample } \\
\text { size }\end{array}$ & Design & $\begin{array}{c}\text { Duration } \\
\text { of study }\end{array}$ & $\begin{array}{c}\text { Duration of } \\
\text { RA }\end{array}$ & Intervention & $\begin{array}{c}\text { Key Efficacy } \\
\text { variable }\end{array}$ & Key finding & Reference \\
\hline $\begin{array}{l}1992- \\
1993\end{array}$ & $\begin{array}{l}\text { Intensive inpatient } \\
\text { multidisciplinary care } \\
\text { versus standard care } \\
\text { (out-patient) }\end{array}$ & 80 & $\begin{array}{l}\text { RCT } \\
2 \text { arms: control ; } \\
\text { intervention care; } \\
\text { site: Hospital based } \\
\text { rheumatology }\end{array}$ & 1 year & $0.5-33$ & $\begin{array}{l}\text { Inpatient training } \\
\text { of exercise, } \\
\text { coping and } \\
\text { occupational } \\
\text { therapy in } \\
\text { interventional arm, } \\
\text { background Std } \\
\text { care (RA) }\end{array}$ & $\begin{array}{l}\text { pain VAS, } \\
\text { Ritchie articular } \\
\text { index, HAQ, } \\
\text { anxiety and } \\
\text { depression scale. }\end{array}$ & $\begin{array}{l}\text { reduced pain, } \\
\text { improved } \\
\text { indices of } \\
\text { function; no } \\
\text { benefit for } \\
\text { anxiety } \\
\text { depression after } \\
12 \text { weeks }\end{array}$ & $\begin{array}{l}\text { Vlieland } \text { et al } \\
(1996)^{24}\end{array}$ \\
\hline $\begin{array}{l}1992- \\
1993\end{array}$ & $\begin{array}{l}\text { Intensive inpatient } \\
\text { multidisciplinary care } \\
\text { versus standard care }\end{array}$ & 80 & $\begin{array}{l}\text { RCT } \\
2 \text { arms: control ; } \\
\text { intervention care; } \\
\text { site: } \\
\text { Hospital based rheumatology }\end{array}$ & 2 year & $0.5-33$ & $\begin{array}{l}\text { Inpatient training } \\
\text { of exercise, } \\
\text { coping and } \\
\text { occupational } \\
\text { therapy in } \\
\text { interventional arm, } \\
\text { Background Std } \\
\text { care (RA) }\end{array}$ & $\begin{array}{l}\text { pain VAS, } \\
\text { Ritchie articular } \\
\text { index, } \\
\text { HAQ, } \\
\text { patient and } \\
\text { physician global } \\
\text { assessment. }\end{array}$ & $\begin{array}{l}\text { no significant } \\
\text { change in } \\
\text { HAQ; } \\
\text { rest significant } \\
\text { improvement }\end{array}$ & $\begin{array}{l}\text { Vlieland et al } \\
\quad(1997)^{23}\end{array}$ \\
\hline 1999 & $\begin{array}{l}\text { Long-term benefit of } \\
\text { multidisciplinary } \\
\text { arthritis training } \\
\text { programme }\end{array}$ & 68 & $\begin{array}{l}\text { Study 1: } \\
\text { Prospective RCT } \\
2 \text { arms: control ; } \\
\text { intervention care; } \\
\text { site: Community based } \\
\text { internal medicine } \\
\text { Study 2: } \\
\text { cross over non- controlled } \\
\text { observation }\end{array}$ & $\begin{array}{l}\text { Study } 1 \text { : } \\
1 \text { year; } \\
\text { Study } 2 \text { : } \\
5 \text { years }\end{array}$ & $\begin{array}{c}0.4 \text { to } 30 \\
\text { years }\end{array}$ & $\begin{array}{l}\text { Multidisciplinary } \\
\text { arthritis training } \\
\text { programme, } \\
\text { background Std } \\
\text { care (RA) }\end{array}$ & $\begin{array}{l}\text { HAQ, } \\
\text { coping, } \\
\text { Beck depression, } \\
\text { knowledge of } \\
\text { drug therapy, } \\
\text { physiotherapy, } \\
\text { joint protection, } \\
\text { relaxation } \\
\text { exercises. }\end{array}$ & $\begin{array}{l}\text { significant } \\
\text { improvement in } \\
\text { all variables in } \\
\text { intervention } \\
\text { arm }\end{array}$ & $\begin{array}{l}\text { Scholten et al } \\
\quad(1999)^{25}\end{array}$ \\
\hline 2003 & $\begin{array}{l}\text { Group } \\
\text { education in } \\
\text { patient of RA and } \\
\text { their partner }\end{array}$ & 218 & $\begin{array}{l}\text { RCT; } \\
3 \text { arms: control } \\
\text { intervention care; } \\
\text { site: } \\
\text { Hospital based communication } \\
\text { studies }\end{array}$ & $\begin{array}{c}12 \\
\text { months }\end{array}$ & $\begin{array}{l}\text { mean } \\
11.7 \pm 9.8 \\
\text { Years }\end{array}$ & $\begin{array}{l}\text { Education } \\
\text { programme, } \\
\text { background Std } \\
\text { care (RA) }\end{array}$ & $\begin{array}{l}\text { Self-efficacy pain, } \\
\text { exercises, } \\
\text { coping, } \\
\text { DAS28, } \\
\text { fatigue -VAS. }\end{array}$ & $\begin{array}{l}\text { improvement in } \\
\text { fatigue and } \\
\text { coping with no } \\
\text { change in rest } \\
\text { variables }\end{array}$ & $\begin{array}{l}\text { Riemsma et al } \\
\quad(2003)^{20}\end{array}$ \\
\hline 2008 & $\begin{array}{l}\text { Life style } \\
\text { management for } \\
\text { arthritis programme } \\
\text { (LMAP) }\end{array}$ & 167 & $\begin{array}{l}\text { Parallel-group RCT; } \\
2 \text { arms: control; } \\
\text { intervention care; } \\
\text { site: } \\
\text { Hospital based rehabilitation } \\
\text { center }\end{array}$ & $\begin{array}{c}12 \\
\text { months }\end{array}$ & $\begin{array}{l}\text { mean } \\
7.34 \pm 6.9\end{array}$ & $\begin{array}{l}\text { LMAP versus } \\
\text { standard self- } \\
\text { management } \\
\text { programme (SP), } \\
\text { Background Std } \\
\text { care (RA) }\end{array}$ & $\begin{array}{l}\text { pain VAS, } \\
\text { self-efficacy, } \\
\text { fatigue, } \\
\text { HAQ, } \\
\text { psychological } \\
\text { status, exercise, } \\
\text { Joint protection, }\end{array}$ & $\begin{array}{l}\text { significant } \\
\text { improvement in } \\
\text { all variables }\end{array}$ & $\begin{array}{l}\text { Hammond et al } \\
\qquad(2008)^{16}\end{array}$ \\
\hline
\end{tabular}


A recent longitudinal study by Vriezekolk et al conducted to describe the development and feasibility of the integration of a

CBT within a multimodal rehabilitation programme for highly distressed patients with rheumatic diseases. The study

concluded that a significant improvement was noticed in physical and psychological functioning, attendance rate and satisfaction (17) [Table 5].

Table 5: Multi modular self-management programme in arthritis

\begin{tabular}{|c|c|c|c|c|c|c|c|c|c|}
\hline Year & $\begin{array}{c}\text { Name of } \\
\text { study }\end{array}$ & Sample size & Design & $\begin{array}{c}\text { Duration } \\
\text { of study }\end{array}$ & $\begin{array}{c}\text { Duration } \\
\text { of RA }\end{array}$ & Intervention & $\begin{array}{c}\text { Key Efficacy } \\
\text { variable }\end{array}$ & Key finding & Reference \\
\hline $\begin{array}{l}1996 \text { to } \\
1997\end{array}$ & $\begin{array}{l}\text { SMART } \\
\text { versus } \\
\text { Usual care } \\
\text { or ASMP in } \\
\text { arthritis }\end{array}$ & $\begin{array}{l}\text { 2 RCT: } \\
1^{\text {st }}-1090 \\
2^{\text {nd }}-341 \\
\text { (RA 60\% } \\
\text { OA 40\%) }\end{array}$ & $\begin{array}{l}2 \text { RCT } \\
\text { Staff involved in } \\
\text { administering was blinded to } \\
\text { participant status } \\
2 \text { arms: control ; } \\
\text { intervention care; } \\
\text { Site: Medical information } \\
\text { system, (ARAMIS) databank } \\
\text { center, and community } \\
\text { based medicine }\end{array}$ & $\begin{array}{l}18 \text { months } \\
\text { and } \\
3 \text { years }\end{array}$ & NA & $\begin{array}{l}\text { SMART } \\
\text { versus } \\
\text { ASMP, } \\
\text { background Std } \\
\text { care }\end{array}$ & $\begin{array}{l}\text { HAQ index, } \\
\text { pain VAS, } \\
\text { depression, } \\
\text { role function, } \\
\text { doctor visits, } \\
\text { self-efficacy }\end{array}$ & $\begin{array}{l}\text { improvement in all } \\
\text { baseline variables for } \\
\text { SMART and ASMP } \\
\text { groups with higher } \\
\text { benefit in self efficacy } \\
\text { and doctor visit rate }\end{array}$ & $\begin{array}{l}\text { Lorig et al } \\
(2004)^{30}\end{array}$ \\
\hline $\begin{array}{l}2002 \text { to } \\
2003\end{array}$ & $\begin{array}{l}\text { ASMP } \\
\text { versus } \\
\text { CDSMP in } \\
\text { arthritis }\end{array}$ & $\begin{array}{l}355 \\
\text { RA } 18 \% \text { vs } 13 \% \text {, } \\
\text { OA } 75.7 \% \text { vs } 5.1 \% \text {, } \\
\text { Other arthritis } \\
10.5 \% \text { vs } 19.8 \% \\
\text { in ASMP and CDSMP } \\
\text { groups respectively }\end{array}$ & $\begin{array}{l}\text { RCTs } \\
2 \text { arms: control ; } \\
\text { intervention care; } \\
\text { Site: } \\
\text { Community-based medicine }\end{array}$ & 1 year & NA & $\begin{array}{l}\text { ASMP } \\
\text { versus } \\
\text { CDSMP, } \\
\text { background Std } \\
\text { care }\end{array}$ & $\begin{array}{l}\text { disability, } \\
\text { global health, } \\
\text { pain, fatigue, } \\
\text { exercise, self- } \\
\text { efficacy, } \\
\text { healthcare } \\
\text { utilization }\end{array}$ & $\begin{array}{l}\text { lesser disability } \\
\text { indices, pain, fatigue } \\
\text { and healthcare } \\
\text { utilization with } \\
\text { improved self-efficacy } \\
\text { seen in ASMP group }\end{array}$ & $\begin{array}{l}\text { Lorig et al } \\
(2005)^{31}\end{array}$ \\
\hline 2009 & $\begin{array}{l}\text { ASMP in } \\
\text { arthritis }\end{array}$ & $\begin{array}{l}124 \\
\text { (RA 59, OA 59, } \\
\text { Other arthritis 6) }\end{array}$ & $\begin{array}{l}\text { Cross-sectional } \\
\text { Follow-up } \\
2 \text { arms: control; } \\
\text { intervention care; } \\
\text { Site: Community based health } \\
\text { and life Sciences }\end{array}$ & 8 years & $\begin{array}{l}\text { mean } \\
19 \pm 11 \\
\text { years }\end{array}$ & $\begin{array}{l}\text { ASMP, } \\
\text { background Std } \\
\text { care }\end{array}$ & $\begin{array}{l}\text { self-efficacy, } \\
\text { exercise, } \\
\text { communication } \\
\text { with physician, } \\
\text { HAQ, HADS, } \\
\text { GP visit }\end{array}$ & $\begin{array}{l}\text { Significant } \\
\text { improvement, in all } \\
\text { variables }\end{array}$ & $\begin{array}{c}\text { Barlow et al } \\
(2009)^{32}\end{array}$ \\
\hline
\end{tabular}


Yousefi et al

\begin{tabular}{|c|c|c|c|c|c|c|c|c|c|}
\hline 2005 & $\begin{array}{l}\text { Effect of } \\
\text { education on } \\
\text { perceived } \\
\text { self-efficacy } \\
\text { in arthritis }\end{array}$ & $\begin{array}{l}80 \\
\text { OA (24 vs 16) } \\
\text { RA }(9 \text { v } 7) \\
\text { Other }(7 \text { vs } 17) \\
\text { in intervention and } \\
\text { control group } \\
\text { respectively }\end{array}$ & $\begin{array}{l}\text { Pre-test and post-test } \\
\text { equivalent control group } \\
2 \text { arms: control; } \\
\text { intervention care; } \\
\text { Site: } \\
\text { Hospital based } \\
\text { health sciences }\end{array}$ & 6 months & $\begin{array}{l}6 \text { months } \\
\text { to more } \\
\text { than } 11 \\
\text { years }\end{array}$ & $\begin{array}{l}\text { Education } \\
\text { programme- } \\
\text { arthritis, } \\
\text { treatment, care, } \\
\text { preventing } \\
\text { method, exercise. } \\
\text { background Std } \\
\text { care }\end{array}$ & $\begin{array}{l}\text { Self-efficacy in } \\
\text { pain, } \\
\text { ASES }\end{array}$ & $\begin{array}{l}\text { significant } \\
\text { improvement, in all } \\
\text { variables }\end{array}$ & $\begin{array}{l}\text { Unsal.and } \\
\text { Kasikei. } \\
(2009)^{22}\end{array}$ \\
\hline $\begin{array}{c}2008 \text { to } \\
2009\end{array}$ & $\begin{array}{l}\text { CBT with } \\
\text { multimodal } \\
\text { rehabilitation } \\
\text { in } \\
\text { Rheumatic } \\
\text { disease }\end{array}$ & $\begin{array}{l}25 \\
\text { (Inflammatory } \\
\text { rheumatic disease } \\
\text { and OA) }\end{array}$ & $\begin{array}{l}\text { proof-of-concept study } \\
2 \text { arms: control; } \\
\text { intervention care; } \\
\text { Site: } \\
\text { Clinic rheumato-logy based } \\
\text { rheumatology }\end{array}$ & 15 months & NA & $\begin{array}{l}\text { Cognitive } \\
\text { behaviour therapy } \\
\text { with multimodal } \\
\text { rehabilitation } \\
\text { programme. } \\
\text { background Std } \\
\text { care }\end{array}$ & $\begin{array}{l}\text { significant } \\
\text { improvement in } \\
\text { physical and } \\
\text { psychological } \\
\text { functioning, } \\
\text { attendance rate, } \\
\text { satisfaction }\end{array}$ & $\begin{array}{l}\text { significant } \\
\text { improvement in all } \\
\text { variables. }\end{array}$ & $\begin{array}{l}\text { Vriezekolk } \\
\quad \text { et al } \\
(2012)^{17}\end{array}$ \\
\hline
\end{tabular}


A study by Shadick et al which conducted to proof-of-concept of psychotherapeutic intervention on disease activity and psychological status in RA. The study results indicated that the intervention model was feasible and acceptable, provide some sustainable benefits to patients and may complement medical management of the disease (18) [Table 1]. These results suggest that CBT can be an effective adjunctive treatment for patients with RA and can help them for both psychological and physical morbidity.

\section{Education}

A study by Hill et al demonstrated improvement in drug adherence with an education programme (19) [Table 1]. Another study by Riemsma et al designed to determine the effects of group education. The study finds indicated that participation of a significant other in psychoeducational programmes does not have only positive effects. Instead of stimulating patients to adopt beneficial health behaviours and increase their self-efficacy expectations, participation of a significant other led to decreases in self-efficacy and increased fatigue, whereas patients participating in group education without partners showed increases in self-efficacy and decreased fatigue (20) [Table 2]. A study by Savelkoul et al conducted to assess the effects of a coping intervention group (CIG), (Fig. 2) and the manual support control group (MSCG), [Fig. 2] with rheumatic diseases. The results show that, at post-intervention, the coping intervention resulted in more action-directed coping than in the mutual support groups (21) [Table 3]. 
Table 3: Uni-modular self-management programme in arthritis

\begin{tabular}{|c|c|c|c|c|c|c|c|c|c|}
\hline Year & $\begin{array}{l}\text { Name of } \\
\text { study }\end{array}$ & Sample size & Design & $\begin{array}{c}\text { Duration } \\
\text { of study }\end{array}$ & $\begin{array}{c}\text { Duration } \\
\text { of RA }\end{array}$ & Intervention & $\begin{array}{c}\text { Key } \\
\text { efficacy } \\
\text { variable }\end{array}$ & Key finding & Reference \\
\hline 2001 & $\begin{array}{l}\text { Effect of } \\
\text { coping } \\
\text { intervention } \\
\text { in rheumatic } \\
\text { diseases }\end{array}$ & \begin{tabular}{l}
\multicolumn{1}{c}{168} \\
RA $67 \%$, \\
OA3.6\% \\
and \\
RA54.5\%, \\
OA9.1\% \\
And RA55.4\% \\
OA10.7\% \\
in CIG and \\
MSCG and \\
WLCG \\
respectively
\end{tabular} & $\begin{array}{l}\text { Single } \\
\text { Blind } \\
\text { (assessor) } \\
\text { RCT } \\
3 \text { arms: } \\
\text { control; } \\
\text { intervention } \\
\text { care; } \\
\text { Site: } \\
\text { Hospital } \\
\text { based health } \\
\text { education }\end{array}$ & $\begin{array}{c}6 \\
\text { months }\end{array}$ & $\begin{array}{c}\text { Mean were } \\
12.6 \pm 10.75\end{array}$ & $\begin{array}{l}\text { CIG } \\
\text { programme: } \\
\text { versus } \\
\text { MSCG } \\
\text { programme, } \\
\text { background } \\
\text { Std care }\end{array}$ & $\begin{array}{l}\text { coping, } \\
\text { functional, } \\
\text { health } \\
\text { status, } \\
\text { life } \\
\text { satisfaction }\end{array}$ & $\begin{array}{l}\text { CIG showed } \\
\text { better } \\
\text { improvement } \\
\text { in coping } \\
\text { and } \\
\text { functional } \\
\text { state than } \\
\text { other arms }\end{array}$ & $\begin{array}{c}\text { Savelkoul } \\
\text { et al } \\
(2001)^{21}\end{array}$ \\
\hline
\end{tabular}

A study by Unsal and Kasikei conducted to determine the effects of education on self-efficacy perception of arthritis individuals. Study finds indicated that self-efficacy levels after education were significantly improved in the experimental group (22) [Table 5].

Few studies have explored the effect of patient education (PE) in RA patients, but seem it is an essential part of the successful management of RA disease.

\section{Multidisciplinary programme}

Two studies of Vlieland et al designed to compare the effects of in-patient multidisciplinary treatment with standard outpatient care. The results showed that percentage of patients responding to the ACR criteria was significantly greater in the in-patient group $(23,24)$ [Table 2]. Scholten et al conducted two studies to assess the sustainable benefits of a professional, multidisciplinary training programme for RA patients. Results of the one year study and five years after baseline evaluation indicated significant improvements in HAQ, depression decreased, reduction in fatigue, increased in coping capacity and knowledge of drug 
therapy, sustained improvement in physiotherapy focussed on joint protection and relaxation exercises (25) [Table 2]. The review of studies demonstrated that in a Rheumatology clinic with multidisciplinary team-care has a beneficial effect with respect to disease activity and emotional status. Therefore, a multifactorial approach utilizing medications and self-management techniques is necessary. Fortunately, under proper counselling, individuals with rheumatoid arthritis can safely exercise, improving overall physical fitness, greater ease with activities of daily living and an improved sense of wellbeing.

\section{Self-management}

A longitudinal community based study by Lorig et al was designed to compare three- and sixweek same ASMP. The results indicated that the three week ASMP intervention (Fig. 2) was not as effective in changing health behaviours, health status, or healthcare utilization as the six-weeks ASMP (26) [Table 4]. 
Table 4: Multi-modular self-management programme in arthritis

\begin{tabular}{|c|c|c|c|c|c|c|c|c|c|}
\hline Year & $\begin{array}{c}\text { Name of } \\
\text { study }\end{array}$ & Sample size & Design & $\begin{array}{l}\text { Duration } \\
\text { of study }\end{array}$ & $\begin{array}{c}\text { Duration } \\
\text { of RA }\end{array}$ & $\begin{array}{c}\text { Interven } \\
\text { tion }\end{array}$ & $\begin{array}{c}\text { Key Efficacy } \\
\text { variable }\end{array}$ & $\begin{array}{c}\text { Key } \\
\text { finding }\end{array}$ & Reference \\
\hline 1998 & $\begin{array}{l}\text { Patient } \\
\text { education } \\
\text { of ASMP } 6 \\
\text { week } \\
\text { versus } 3 \\
\text { week } \\
\text { version }\end{array}$ & $\begin{array}{l}151(14 \% \text { RA, } \\
56 \% \text { OA, } \\
\text { Other } 19 \%)\end{array}$ & $\begin{array}{l}\text { Comparison study } \\
2 \text { arms: control ; } \\
\text { intervention care; } \\
\text { Site: } \\
\text { Community based } \\
\text { patient education }\end{array}$ & 4 months & NA & $\begin{array}{l}\text { ASMP } 6 \\
\text { week } \\
\text { module } \\
\text { versus } 3 \\
\text { week } \\
\text { module, } \\
\text { backgrou } \\
\text { nd Std } \\
\text { care }\end{array}$ & $\begin{array}{l}\text { pain VAS, } \\
\text { disability, } \\
\text { depression, } \\
\text { exercise, } \\
\text { physician visit }\end{array}$ & $\begin{array}{l}6 \text { week } \\
\text { ASMP } \\
\text { better than } \\
3 \text { week } \\
\text { ASMP }\end{array}$ & $\begin{array}{l}\text { Lorige } \text { et } \\
\text { al } \\
(1998)^{26}\end{array}$ \\
\hline 1998 & $\begin{array}{l}\text { Long-term } \\
\text { outcome of } \\
\text { ASMP in } \\
\text { arthritis }\end{array}$ & $\begin{array}{l}112 \text { (RA } 46 \% \\
\text { OA } 44 \% \\
\text { Other } 10 \% \text { ) }\end{array}$ & $\begin{array}{l}\text { Pre - test } \\
\text { post - test } \\
1 \text { arms } \\
\text { intervention care; } \\
\text { Site: } \\
\text { Community- } \\
\text { based } \\
\text { psychological } \\
\text { rheumatology }\end{array}$ & $\begin{array}{c}12 \\
\text { months }\end{array}$ & $1-49$ years & $\begin{array}{l}\text { ASMP. } \\
\text { back } \\
\text { grand } \\
\text { Std care }\end{array}$ & $\begin{array}{l}\text { Self-efficacy } \\
\text { pain, } \\
\text { exercise, } \\
\text { communication } \\
\text { with physician, } \\
\text { HAQ, } \\
\text { pain VAS, } \\
\text { HADS, } \\
\text { GP visit }\end{array}$ & $\begin{array}{l}\text { significant } \\
\text { improvem } \\
\text { ent in all } \\
\text { variables }\end{array}$ & $\begin{array}{l}\text { Barlow et } \\
\text { al }(1998)^{27}\end{array}$ \\
\hline 1999 & $\begin{array}{l}\text { Arthritis } \\
\text { self- } \\
\text { manager } \\
\text { through an } \\
\text { Adult } \\
\text { education } \\
\text { programme }\end{array}$ & $\begin{array}{l}89 \text { (RA } 45 \% \text {, } \\
\text { OA } 48 \%, \\
\text { Other } 42 \%)\end{array}$ & $\begin{array}{l}\text { Pre-test post- test } \\
1 \text { arms } \\
\text { intervention care; } \\
\text { Site: Health } \\
\text { service network, } \\
\text { arthritic care } \\
\text { network and } \\
\text { community-based } \\
\text { psychological } \\
\text { rheumatology }\end{array}$ & 4 months & $0-50$ & $\begin{array}{l}\text { ASMP, } \\
\text { backgrou } \\
\text { nd Std } \\
\text { care }\end{array}$ & $\begin{array}{l}\text { MHAQ, } \\
\text { pain VAS, } \\
\text { fatigue VAS, } \\
\text { depression, self- } \\
\text { efficacy for pain } \\
\text { VAS, exercise, } \\
\text { communication } \\
\text { with physician, }\end{array}$ & $\begin{array}{l}\text { significant } \\
\text { improvem } \\
\text { ent in all } \\
\text { variables }\end{array}$ & $\begin{array}{l}\text { Barlow et } \\
\text { al }(1999)^{28}\end{array}$ \\
\hline 2000 & $\begin{array}{l}\text { ASMP in } \\
\text { arthritis } \\
\text { in UK }\end{array}$ & $\begin{array}{l}544 \\
\text { RA37\% vs } \\
33 \% \\
\text { OA52\% vs } \\
52 \% \\
\text { Other11\% vs } \\
14 \% \text { in } \\
\text { intervention } \\
\text { and control } \\
\text { groups, } \\
\text { respectively }\end{array}$ & $\begin{array}{l}\text { Pragmatic RCT } \\
2 \text { arms: control ; } \\
\text { intervention care; } \\
\text { Site: } \\
\text { Community- } \\
\text { based } \\
\text { psychological } \\
\text { rheumatology }\end{array}$ & $\begin{array}{c}16 \\
\text { months }\end{array}$ & $\begin{array}{l}\text { Mean } \\
10.7 \pm 11.2 \\
\text { versus } \\
11.3 \pm 10.9 \\
\text { interventio } \\
\mathrm{n} \text { and } \\
\text { control } \\
\text { groups }\end{array}$ & $\begin{array}{l}\text { ASMP, } \\
\text { backgrou } \\
\text { nd Std } \\
\text { care } \\
\text { (RA) }\end{array}$ & $\begin{array}{l}\text { exercises, diet } \\
\text { communication } \\
\text { with physician, } \\
\text { MHAQ, } \\
\text { pain VAS, } \\
\text { HADS }\end{array}$ & $\begin{array}{l}\text { significant } \\
\text { improvem } \\
\text { ent in all } \\
\text { variables }\end{array}$ & $\begin{array}{l}\text { Barlow et } \\
\text { al }(2000)^{29}\end{array}$ \\
\hline
\end{tabular}

Barlow et al has conducted a study to determine the longterm effect of ASMP in physical and psychological wellbeing in patient suffering from arthritis. Data was collected via mailed selfadministered questionnaires mailed. The findings of the study had demonstrated that patient 
with arthritis derives substantial and prolonged benefits in terms of perceived ability to manage arthritis, reduction in pain and improved physical and psychological wellbeing (27) [Table 4].

Another same design study by Barlow et al did to determine Arthritis self-management Programme (ASMP) is effective when delivered in an adult education setting. The finding of the study showed the ASMP programme was not only acceptable to people with arthritis, but can offer substantial benefits in terms of an enhanced sense of control, a reduction in pain, increased use of cognitive and behavioural techniques, and perceived ability to discuss arthritis in health settings (28) [Table 4]. In 2000, Barlow et al conducted a study to determine whether the ASMP improves perceptions of control, health behaviours and health status and changes use of healthcare resources. Participants were given a copy of the Arthritis help book. The ASMP had a significant effect on arthritis self-efficacy for other symptoms and pain subscales, health behaviours, less depressed and had greater positive mood (29) [Table 4]. Lorig et al tried to examine two studies of the self-management, arthritis relief therapy (SMART), (Fig. 2) and to compare it with the ASMP. Participants were recruited from the arthritis, rheumatism, and aging, medical information system (ARAMIS) databank centers. The finding of the study indicated that a mail-delivered arthritis self-management programme, SMART, was similarly effective in the classic ASMP, with slightly better results in the first year and a slightly more rapid attenuation over the next two years (30) [Table 5]. Lorig et al conducted a study to compare the relative effectiveness of the ASMP and the generic chronic disease selfmanagement programme (CDSMP), (Fig. 2) and background standard care for individuals with arthritis. The finding results showed both programmes had positive effects, and the CDSMP should be considered a viable alternative (31) [Table 5]. 
In 2009, Barlow et al conducted a study to examine the pattern of scores on selfefficacy, health status and use of self-management techniques among a group of ASMP participants. Results showed that long-term maintenance of self-efficacy, psychological wellbeing and self-management techniques may be possible following attendance on the ASMP (32) [Table 5]. Published literature suggests that patients with RA will need long term care. The treatment comprises of not only drug therapy, but also a self-management programme of educational instruction, guidance and support to cope up with the consequences of the disease and manage this persistent, damaging, inflammatory disorder.

\section{Physical therapy (exercise)}

An empirical study by Bell et al conducted to evaluate the short term efficacy of a Physical therapy (PT) programme (Fig. 2) for people with RA. Improvement was noted in primary variables of self-efficacy, disease management knowledge, duration of morning stiffness and tender joint count, but not significantly change for the pain VAS and disease status measures (33) [Table 1]. Until now, the short-term and long-term health benefits and risks of physical activity at moderate and vigorous intensity levels have not yet been compared in early or established RA.

\section{Dietary interventions}

Several clinical trials, studies conducted to assess the effect of dietary interventions in RA (34-39) [Table 1]. The findings also showed that dietary interventions can assist with the management of disease symptoms that accompany rheumatoid arthritis (RA), such as pain, tender swollen joints, stiffness and associated disability and disease progression. Clinical trials 
demonstrated that a subset of patients will benefit from following a vegetarian, vegan, or Mediterranean-style diet, or by eliminating certain foods from their diet (40). This type of diet has shown to be associated with anti-inflammatory effects $(30,31,41)$ which are of desired benefit in RA. Mahan et al suggested the dietary changes to promote an anti-inflammatory diet (42). All studies of diet and RA were carried on an established and prolong disease. Dietary therapy is an area of self-help which RA patients frequently want to explore, typically in the early stages of disease, but little is known about the extent of dietetic involvement in rheumatology. Good nutrition is an essential part of RA self-care because without it the body and sometimes medications does not work as well they be.

\section{DISCUSSION}

Lifelong disability, excruciating pain, psychological fatigue, anxiety and decline in life quality are consequences have always been associated with RA (43). Aggressive therapies with potent drugs like biologics are very effective but fraught with dangers of serious drug toxicity and life threatening adverse events $(4,9,10,13)$. It was observed that despite excellent advances in management care, patients suffering from RA continue to develop functional limitations, deformities $(9,10)$. It is against this background that more evidence of the efficacy of adjunct therapy with non-pharmacological methods (exercise, diet, cognitive education) be obtained through well designed studies in RA.

The concept of early and aggressive treatment of RA has occupied center stage since 1989, when reversing the pyramid (the step-down bridge concept) was published (44). From the year 1990, there was an increasing awareness about the concept "a window of opportunity for 
the therapeutic consultations/treatment in rheumatoid arthritis" $(45,46)$. Experimental methods were used to find a solution for "how can we meet the patient's need in the best way?" (47).

Three early period experimental approaches demonstrated the path of the required change in non-pharmacological treatment measures. The first among the three was the arthritis self-management Programme (ASMP), is the prototype arthritis education programme.

Originally developed by Lorig (1981) at Stanford University, USA. The programme was adopted by the arthritis foundation in 1981 (48). The next one was based on visits carried out by groups of doctors to their patients, and was created by Dr John Scott in 1997 (49). The third experimental programme focussed on patients, staying far away from city centers in remote areas and access for medical attention was enhanced using the telephone or by other electronic means like the Internet (49-51).

Non-drug therapies are often employed as complementary adjuncts to pharmacological treatment to aid coping mechanism of RA patients as they combat the disease (52). Several nonpharmacological clinical experimental methods and or models such as ASMP or ASHC (28), CBT (14), LMAP (16), SMART (30) and CDSMP (31) were evaluated in patients suffering from arthritis. Table 2, Table 4 and Table 5 describe the key features of several studies undertaken to evaluate such modalities of treatment. These studies have demonstrated the role of a self-management programme in RA.

Using a multi-modular self-management programme for patients, Vlieland (1997) showed improvement of PHGH (physician global health) and PTGL (patient global health) by intensive inpatient multidisciplinary care versus standard care (24). Several studies using a modular multi programme (Tables 2, 4 and Table 5) made similar observations in different 
groups of subjects, and noted a significant decrease in pain VAS and physician visit times or healthcare utilization (Tables 4 and 5).

The effectiveness of multidisciplinary teamcare in countering established RA is well recognized. The benefits of such non-pharmacological treatments can be gauged from the conclusions drawn from several RCTs and Cochrane Reviews. It is hardly therefore surprising to note that RA patients constitute the high end spectrum of complementary and alternative practice (CAP) therapies given the nature of the disease as particularly chronic and persistent (43). A diverse range of modalities comprising the "multidisciplinary Rheumatology team", as various health professionals prefer to label it, includes exercise therapy, physical modalities, orthoses and assistive devices, self-management and dietary interventions as constituent members. Recent years have witnessed the publication of a sizeable number of reviews focussed on a broad spectrum of non-pharmacological interventions in RA $(53,54)$.

Contemporary interventions that supplement pharmacological and surgical interventions include conventional therapies like physical therapy and occupational therapy, besides rehabilitation and self-management programmes are put to use (43). Evidence strongly suggests that joint specific dynamic exercises may lead to a significant amplification in strength and physical function (55). The beneficial effects of self-management and functional ability through occupational therapy have shown in recent meta-analyses $(56,57)$. Similarly, employment of hydrotherapy in rheumatoid arthritis cases has also reported positive results $(58,59)$. There have been insufficient studies on the effectiveness of specific diets in managing RA Efficacy with diet modification has been noted by several RCTs which, for example, noted that the vegetarian diet group exhibited considerably better effects on pain and indices of disease activity and across most of clinical variables $(36,60,61)$. Is investigating a range of diets, 
including gluten free, vegetarian, vegan and fasting diets. However, long term compliance and nutritional deficiencies, reduced the acceptability of many dietary interventions (Table 1).

Self-management programme is reported to impart psychological courage, able to face the lifelong challenge of RA and increases the acceptability of medical treatment (62). Management should address both the physical and mental aspects of life (63). Further, selfmanagement programme provides a major support to the patient (49). It also provides better awareness of the ailment and thereby enhances the confidence to self-manage health problems with great courage $(15,64)$. It also encourages better compliance of the drug management programme, regular assessment and monitoring of disease and drug related effects, to know the progress and goal setting for the treatment and achieving targets in the set time (65). The first aim of a combined strategy with supervised sustained standard of care medical management and self-management programme is to obtain an early remission of the disease. The next target is to bring the body to a fully functional status and resume work with great physical and mental involvement and all this may also require vocational rehabilitation (65). Rheumatoid arthritis self-management intervention programmes help people to maximize their abilities and reduce pain, functional limitations, disability, depression and in increasing self-care behaviours (48). A self-management intervention programme to provide the knowledge, skills and confidence to manage rheumatoid arthritis was devised (66).

The very few rheumatologists available in developing countries do not have the time and the inclination for community and patient education. Education should be left to the allied rheumatology health professionals if available (67). A professional, multidisciplinary approach to educate patients with rheumatoid arthritis leads to a significant and sustained improvement of the clinical outcome and is an approach that should be established as part of conventional 
therapy (25). The members of a multidisciplinary team should take particular care to ensure that a common approach to patient education is arrived at such that the patients receive a consistent health message (68).

In the literature review studies have shown that a short period of initial intensive nonpharmacological therapy (mostly cognitive behaviour change and supervised exercises) is followed by long term gains in the form of better improvement in RA symptoms and control and better health measures (Tables 1, 2, 4 and Table 5). A number of multi modular programmes in patient of RA or other arthritis, have made similar observations in different groups of subjects (Tables 2, 4 and Table 5). Physical therapy and exercise showed significant reduction in fatigue and significant improvement in joint protection, communication with physicians, and dietary habits. A number multi modular intervention programme (Tables 2, 4 and Table 5) made similar observations in different groups of subjects in patient of RA or other arthritis.

The literature on the effectiveness of non-pharmacological treatment modalities in RA, ie the available studies support the use of dynamic exercise and cognitive behavioural interventions, and to a lesser extent of joint protection programmes and foot orthoses and dietary manipulation. Decreased physical activity levels in RA patients combined with the symptoms of the disease eg pain and fatigue corroborate to formulate a vicious circle that contributes directly to a detrimental effect on other aspects of skeletal muscle health (69).

Rheumatoid arthritis patients consistently report a $70 \%$ reduction in strength in comparison to healthy counterparts (70). Two years be strengthening programmes entailed significant improvements in subjective patient assessments of disability by the HAQ (71). Decreased habitual physical activity in patients with RA is generally attributed to joint pain, 
restricted mobility, fatigue, reduced muscle mass, strength and endurance (72). A corroboration of aerobic and strength exercises training constitutes an ideal exercise programme for treatment of RA patients (73). Exercise is an important component of several self-management programmes and several studies are reviewed and described in the above section on selfmanagement progress. Research has validated the beneficial role of exercise in yielding specific health benefits in people with RA.

As demonstrated by previous research, exercise is fundamentally beneficial for RA patients when a comparison of the effectiveness of high and low intensity exercise training in stable RA is undertaken. The intervention programme on the improvement of life quality in RA patients is multifaceted and there are other modes of non-pharmacological treatment of RA same occupational therapy. My best knowledge it is not available RCT study base on decreasing pain with big sample size, especially in only RA till date in these fields is included.

Rheumatoid arthritis patients are often treated aggressively with disease modifying anti rheumatic drugs (DMARD) [eg Methotrexate, hydroxychloroquin etc] along with steroids in addition to analgesics and other anti-inflammatory drugs and supportive therapy (4). Such a therapy is fraught with drug related side effects and toxicity and more so as RA patients need long term treatment $(9,10,13)$. There is a need to quickly control the disease and judiciously reduce the medicines to the minimum to sustain control. This is not an easy task and in real life continues to be a major therapeutic challenge. In the last decade or so, biologic DMARDs have emerged as very potent medications but again are extremely expensive and with a potential risk of several side effects including proneness for tuberculosis and life threatening infections $(4,13)$. 
Gentler treatment modalities are required to augment the therapeutic efficacy of anti RA medications. A non-pharmacological treatment could fill-up this void and also improve the side-effect profile during this period.

In conclusion epidemiology has taken on new roles in the management of healthcare services. The literature on the effectiveness of non-pharmacological treatment modalities in RA, ie the available studies support the use of dynamic exercise and cognitive behavioural interventions, and to a lesser extent of joint protection programmes and foot orthoses and dietary manipulation. The effectiveness of multidisciplinary team-care in countering established RA is well recognized. It was indentured that despite excellent advances in management care, patients suffering from RA continue to develop functional limitations, deformities. Contemporary computation of the provision and reimbursement of healthcare is based on existing evidence.

Policy direction, formulates and purchase are regulated by the requirement and supply of data on the effective parameters of interventions. On similar note researchers, healthcare professionals and patients also subscribe to the informational paradigm to identify and define research parameters, and improve upon existing clinical practices and self-management stratification. Self-management, arthritis programmes, though found useful by several studies described above are uncommon in routine clinical practice. Therefore, it is prudent to consider non-pharmacological methods adjunct to standard care with drugs. At this stage, it may be advisable for patients to also opt for an adjunct non-drug treatment like the patients with counselling alongside the standard of care (53). These findings have implications for health policy, and allocation of funding for both healthcare and research. Ethnicity, presumably, may play a major role in the extent of coping with pain and arthritis. Non-pharmacological means to 
improve the management of RA, as an adjunct therapy to standard of care, holds a socioeconomic appeal for the community. Our results can be exploited further by constructing preventive instructional non-pharmaceutical strategies to treat RA that is suited to the community.

\section{Limitation of the literature review}

Some databases might not have covered due to lack of access to them. Therefore, it is not claimed that this review covers all relevant articles. Occasionally original research articles are cited without a clear account of how articles were found. At some places personal experience and conventional wisdom are included which may be difficult to distinguish.

\section{ACKNOWLEDGMENT}

This literature review was supported by Deputy for Medical Education and Deputy for Research, Hormozgan University of Medical Sciences, Bandar Abbas, Iran and the Center for Rheumatic diseases (CRD) Pune, Interdisciplinary School of Health Sciences University of Pune, India. We thank the support and contributions of doctors, colleagues and staff in ARCF CRD Pune and community rheumatology clinic Bandar Abbas, Iran. The following experts participated in the development of the study, I would like to acknowledge the contribution and dedicated work of the late Associate Professor Azim Nejatizade, Dr Anuradha Venugopalan. 


\section{REFERENCES}

1. Chopra A, Abdel-Nasser AM. Epidemiology of rheumatic musculoskeletal disorders in the developing world. Best Practice Res Clin Rheumatol 2008; 22: 583-604.

2. Oliver JE, Silman AJ. What epidemiology has told us about risk factors and aetiopathogenesis in rheumatic diseases? Arthritis Res Ther 2009; 11: 223.

3. Wigley R, Chopra A, Wigley S, Akkoyunlu-Wigley A. Does control of rheumatic disease raises the standard of living in developing countries? Clin Rheumatol 2009; 28: $621-2$.

4. Chopra A, Shankar S. Biosimilar DMARD in Rheumatology: A general perspective with focus on India. Indian J Rheumatol 2012; 7: 89-96.

5. Davatchi F, Jamshidi A-R, Tehrani Banihashemi A, Gholami J, Hossein Forouzanfar M, Akhlaghi $M$ et al WHO-ILAR COPCORD Study (Stage 1, urban study) in Iran. J rheumatol 2008; 35: 1384-90.

6. Davatchi F, Jamshidi A-R, Tehrani Banihashemi A, Gholami J, Hossein Forouzanfar M, Akhlaghi M et al. Effect of ethnic origin (Caucasians versus Turks) on the prevalence of rheumatic diseases: a WHO-ILAR COPCORD urban study in Iran. Clin Rheumatol 2009; 28: 1275-82.

7. Davatchi F. Rheumatic diseases in the APLAR region. APLAR J Rheumatol 2006; 9: $5-10$.

8. Symmons D, Turner G, Webb R, Asten P, Barrett E, Lunt M et al. The prevalence of rheumatoid arthritis in the United Kingdom: new estimates for a new century. Rheumatol 2002; 41: 793-800. 
9. Scott DL, Smith C, Kingsley G. What are the consequences of early rheumatoid arthritis for the individual? Best Practice Res Clin Rheumatol 2005; 19: 117-36.

10. Symmons D, Tricker K, Harrison M, Roberts C, Davis M, Dawes P et al. Patients with stable long-standing rheumatoid arthritis continue to deteriorate despite intensified treatment with traditional disease modifying anti-rheumatic drugs-results of the British Rheumatoid Outcome Study Group randomized controlled clinical trial. Rheumatoid Oxford, 2006; 45: 558-65.

11. Chopra A. Community rheumatology in India: a COPCORD driven perspective. Indian J Rheumatol 2009; 4: 119-26.

12. Looper KJ, Mustafa SS, Zelkowitz P, Purden M, Baron M. Work instability and financial loss in early inflammatory arthritis. Int J Rheum Dis 2012; 15: 546-53.

13. Kelley WN. Kelley's textbook of rheumatology. Elsevier Health Sciences 2012; 13-56.

14. Sharpe L, Sensky T, Timberlake N, Ryan B, Brewin C, Allard S. A blind, randomized, controlled trial of cognitive-behavioral intervention for patients with recent onset rheumatoid arthritis: preventing psychological and physical morbidity. Pain 2001; 89: 275-83.

15. Sharpe L, Sensky T, Timberlake N, Ryan B, Allard S. Long-term efficacy of a cognitive behavioural treatment from a randomized controlled trial for patients recently diagnosed with rheumatoid arthritis. Rheumatoid 2003; 42: 435-41.

16. Hammond A, Bryan J, Hardy. A Effects of a modular behavioral arthritis education program: a pragmatic parallel-group randomized controlled trial. Rheumatol 2008; 47: $1712-18$. 
17. Vriezekolk JE, Geenen R, van den Ende CH, Slot H, van Lankveld WG, van Helmond T. Behavior change, acceptance, and coping flexibility in highly distressed patients with rheumatic diseases: feasibility of a cognitive-behavioral therapy in multimodal rehabilitation. Patient Educ Couns 2012; 87: 171-77.

18. Shadick NA, Sowell NF, Frits ML, Hoffman SM, Hartz SA, Booth FD et al. A randomized controlled trial of internal family systems-based psychotherapeutic intervention on outcomes in rheumatoid arthritis: A Proof-of-Concept study. J Rheumatol 2013; 40: 1831-41.

19. Hill J, Bird H, Johnson S. Effect of patient education on adherence to drug treatment in rheumatoid arthritis: a randomized controlled trial. Ann Rheum Dis 2001; 60: 869-75.

20. Riemsma RP, Taal E, Rasker JJ. Group education for patients with rheumatoid arthritis and their partners. Arthritis Care Res 2003; 49: 556-66.

21. Savelkoul M, de Witte LP, Candel MJ, van der Tempel H, van den Borne B. Effects of a coping intervention on patients with rheumatic diseases: results of a randomized controlled trial. Arthritis Care Res 2001; 45: 69-76.

22. Ünsal A, Kasikçi MK. Effect of education on perceived self-efficacy for individuals with arthritis. Int J Caring Sci 2010; 3: 3-11.

23. Vlieland TV, Breedveld F, Hazes J. The two-year follow-up of a randomized comparison of in-patient multidisciplinary team care and routine out-patient care for active rheumatoid arthritis. Rheumatol 1997; 36: 82-5.

24. Vljeland TV, Zwinderman A, Vandenbroucke J, Breedveld F, Hazes J. A randomized clinical trial of in-patient multidisciplinary treatment versus routine out-patient care in active rheumatoid arthritis. Rheumatol 1996; 35: 475-82. 
25. Scholten C, Brodowicz T, Graninger W, Gardavsky I, Pils K, Pesau B et al. Persistent functional and social benefit 5 years after a multidisciplinary arthritis training program. Arch Phys Med Rehabil 1999; 80: 1282-7.

26. Lorig K, González VM, Laurent DD, Morgan L, Laris B. Arthritis self-management program variations: three studies. Arthritis Rheum 1998; 11: 448-54.

27. Barlow J, Turner A, Wright C. Long-term outcome of an arthritis self-management programme. Rheumatol 1998; 37: 1315-19.

28. Barlow JH, Williams B, Wright CC. Instilling the strength to fight the pain and get on with life: learning to become an arthritis self-manager through an adult education programme. Health Educ Res 1999; 14: 533-44.

29. Barlow JH, Turner AP, Wright CC. A randomized controlled study of the arthritis selfmanagement programme in the UK. Health Educ Res 2000; 15: 665-80.

30. Lorig KR, Ritter PL, Laurent DD, Fries JF. Long-term randomized controlled trials of tailored-print and small-group arthritis self-management interventions. Med Care 2004; 42: $346-54$.

31. Lorig K, Ritter PL, Plant K. A disease-specific self-help program compared with a generalized chronic disease self-help program for arthritis patients. Arthritis Care Res 2005; 53: 950-57.

32. Barlow J, Turner A, Swaby L, Gilchrist M, Wright C, Doherty M. An 8-yr follow-up of arthritis self-management programme participants. Rheumatol 2009; 48: 128-33.

33. Bell MJ, Lineker S, Wilkins AL, Goldsmith CH, Badley EM. A randomized controlled trial to evaluate the efficacy of community based physical therapy in the treatment of people with rheumatoid arthritis. J Rheumatol 1998; 25: 231-37. 
34. Kjeldsen-Kragh J, Haugen M, Forre Q, Laache H, Malt UF. Vegetarian diet for patients with rheumatoid arthritis: can the clinical effects be explained by the psycho- logical characteristics of the patients? BJ Rheumatol 1994; 33: 569-75.

35. Nenonen MT, Helve TA, Rauma A-L, Hanninen OO. Uncooked, lactobacilli-rich, vegan food and rheumatoid arthritis. BJ Rheumatol 1998; 37: 274-81.

36. Hafström I, Ringertz B, Spångberg A, von Zweigbergk L, Brannemark S, Nylander I et al. A vegan diet free of gluten improves the signs and symptoms of rheumatoid arthritis: the effect on arthritis correlate with a reduction in antibodies to food antigens. Rheumatol 2001; 40: 1175-79.

37. Hagfors L, Nilsson I, Sköldstam L, Johansson G. Fat intake and composition of fatty acids in serum phospholipids in a randomized, controlled, mediterranean dietary intervention study on patients with rheumatoid arthritis. Nut \& Meta 2005 2: 1-11.

38. Galarraga B, Ho M, Youssef HM, Hill A, McMahon H, Hall C et al. Cod liver oil (n-3 fatty acids) as a non-steroidal anti-inflammatory drug sparing agent in rheumatoid arthritis. Rheumatol 2008; 47: 665-69.

39. Elkan CA, Sjöberg B, Kolsrud B, Ringertz B, Hafström I, FrostegÅrd J. gluten-free vegan diet induces decreased LDL and oxidized LDL levels and raised atheroprotective natural antibodies against phosphorylcholine in patients with rheumatoid arthritis: a randomized study. Arthritis Res \& Therapy 2008; 10: 1-8

40. Vitetta L, Coulson S, Schloss J, Beck SL, Allen R, Sali A. Dietary recommendations for patients with rheumatoid arthritis: a review. Nutr Dietary 2012 (Suppl 4) 1-15.

41. Rayman MP, Pattison DJ. Dietary manipulation in musculoskeletal conditions. Best Practice \& Research Clinical Rheumatol 2008; 22: 535-61. 
42. Mahan LK, Raymond JL, Escott-Stump S. Krause's food \& the nutrition care process. Elsevier Health Sciences 2013; 901-19.

43. Astin JA, Beckner W, Soeken K, Hochberg MC, Berman B. Psychological interventions for rheumatoid arthritis: A meta-analysis of randomized controlled trials. Arthritis Care Res 2002; 47: 291-302.

44. Wilske K, Healey L. Remodeling the pyramid--a concept whose time has come. J rheumatol 1989; 16: 565-67.

45. Quinn M, Emery P. Window of opportunity in early rheumatoid arthritis: the possibility of altering the disease process with early intervention. Clin Exp Rheumatol 2003: 21(SUPP 31), S154-S7.

46. Cush JJ. Early rheumatoid arthritis--is there a window of opportunity? J Rheumatol 2007; 80: $1-7$.

47. Wagner EH, Austin BT, Davis C, Hindmarsh M, Schaefer J, Bonomi A. Improving chronic illness care: translating evidence into action. Health Aff (Millwood) 2001; 20: 64-78.

48. Brady TJ, Kruger J, Helmick CG, Callahan LF, Boutaugh ML. Intervention programs for arthritis and other rheumatic diseases. Health Educ Behav 2003; 30: 44-63.

49. Holman H, Lorig K. Patient self-management: a key to effectiveness and efficiency in care of chronic disease. Public Health Rep 2004; 119: 239.

50. Ludman E, Simon G, Grothaus L, Luce C, Markley D, Schaefer J. A pilot study of telephone care management and structured disease self-management groups for chronic depression. Psychiatr Serv 2007; 58: 1065-72. 
51. Weinberger M. Telephone-based interventions in outpatient care. Ann Rheum Dis 1998; 57: 196-97.

52. Vliet Vlieland TP, Pattison D. Non-drug therapies in early rheumatoid arthritis. Best Practice Res Clin Rheumatol 2009; 23: 103-16.

53. Christie A, Jamtvedt G, Dahm KT, Moe RH, Haavardsholm EA, Hagen KB. Effectiveness of non-pharmacological and nonsurgical interventions for patients with rheumatoid arthritis: an overview of systematic reviews. Phys Ther 2007; 87: 1697-715.

54. Vlieland TV. Non-drug care for RA - is the era of evidence-based practice approaching? Rheumatol, Oxford 2007; 46: 1397-404.

55. Brosseau L, Wells GA, Tugwell P, Egan M, Wilson KG, Dubouloz CJ et al. Ottawa panel evidence-based clinical practice guidelines for therapeutic exercises in the management of rheumatoid arthritis in adults. Phys Ther 2004; 84: 934-72.

56. Van Den Ende C, Vliet VT, Munneke M, Hazes J. Dynamic exercise therapy for rheumatoid arthritis: a systematic review. Rheumatol 1998; 37: 677-87.

57. Steultjens E, Dekker J, Bouter L, Van Schaardenburg D, Van Kuyk M, Van den Ende C. Occupational therapy for rheumatoid arthritis: a systematic review. Arthritis Care Res 2002; 47: 672-85.

58. Hammond A, Young A, Kidao R. A randomised controlled trial of occupational therapy for people with early rheumatoid arthritis. Ann Rheum Dis 2004; 63: 23-30.

59. Verhagen AP, Bierma-Zeinstra SMA, Cardoso JR, De Bie RA, Boers M, De Vet HCW. Balneotherapy for rheumatoid arthritis (review). Cochrane Database Syst Rev 2004; 1(CD000518), 1-27. 
60. Kjeldsen-Kragh J, Haugen M, Borchgrevink CF, Laerum E, Eek M, Mowinkel P et al. Controlled trial of fasting and one-year vegetarian diet in rheumatoid arthritis. Lancet 1991; 338: 899-902.

61. Kjeldsen-Kragh J. Rheumatoid arthritis treated with vegetarian diets. Am J clin nutr 1999; 70: 594-600.

62. Ryan S, Hassell A, Dawes P, Kendall S. Control perceptions in patients with rheumatoid arthritis: the impact of the medical consultation. Rheumatol 2003; 42: 135-40.

63. Bruce B, Fries JF, Murtagh KN. Health status disparities in ethnic minority patients with rheumatoid arthritis: a cross-sectional study. J rheumatol 2007; 34: 1475-79.

64. Brady TJ, Murphy L, O'Colmain BJ, Beauchesne D, Daniels B, Greenberg M et al. Peer reviewed: A Meta-analysis of health status, health behaviors, and health care utilization outcomes of the chronic disease self-management program. Prev Chronic Dis 2013; 10.

65. Luqmani R, Hennell S, Estrach C, Birrell F, Bosworth A, Davenport G et al. British society for rheumatology and British health professionals in rheumatology guidelines for the management of rheumatoid arthritis (the first two years). Rheumatol 2006; 45: $1167-69$.

66. Bruce B, Lorig K, Laurent D. Participation in patient self-management programs. Arthritis Care Res 2007; 57: 851-54.

67. Darmawan J. Recommendations from the community oriented program for control of rheumatic disease for data collection for the measurement and monitoring of health in developing countries. Clin Rheumatol 2007; 26: 853-57. 
68. Harrison BJ, Silman AJ, Wiles NJ, Scott DG, Symmons DP. The association of cigarette smoking with the disease outcome in patients with early inflammatory polyarthritis. Arthritis Rheum 2001; 44: 323-30.

69. Häkkinen A. Effectiveness and safety of strength training in rheumatoid arthritis. Curr Opin Rheumatol 2004; 16: 132-37.

70. Pedersen BK, Saltin B. Evidence for prescribing exercises as therapy in chronic disease. Scand J Med Sci Sports 2006; 16(S1): 3-63.

71. Häkkinen A, Sokka T, Kotaniemi A, Hannonen P. A randomized two-year studies of the effects of dynamic strength training on muscle strength, disease activity, functional capacity, and bone mineral density in early rheumatoid arthritis. Arthritis Rheum 2001; 44: 515-22.

72. Plasqui G. The role of physical activity in rheumatoid arthritis. Physiol Behav 2008; 94: $270-75$.

73. de Jong Z, Munneke M, Jansen LM, Ronday K, van Schaardenburg DJ, Brand R, van den Ende $\mathrm{CH}$ et al. Differences between participants and nonparticipants in an exercise trial in adults with rheumatoid arthritis. Arthritis Care Res 2004; 51: 593-600. 
Yousefi et al 
Non-pharmacological Community Intervention in Rheumatoid Arthritis 